\title{
Ultrasonographic evaluation of cervical length and amniotic fluid index as predictor of pregnancy outcome in case of preterm premature rupture of membrane
}

\author{
Prashant Patil, Archana Kumari*, H. P. Anand
}

Department of Obstetrics and Gynecology, VMMC and Safdarjung Hospital, New Delhi, India

\author{
Received: 11 March 2018 \\ Accepted: 17 March 2018 \\ *Correspondence: \\ Dr. Archana Kumari, \\ E-mail: drarchanakri@gmail.com
}

Copyright: () the author(s), publisher and licensee Medip Academy. This is an open-access article distributed under the terms of the Creative Commons Attribution Non-Commercial License, which permits unrestricted non-commercial use, distribution, and reproduction in any medium, provided the original work is properly cited.

\begin{abstract}
Background: Preterm premature rupture of membrane (PPROM) is among the most important cause of the perinatal morbidity and mortality. We sought to determine whether cervical length and amniotic fluid index individually or in combination can predict the pregnancy outcome in cases of PPROM.

Methods: The prospective observational study was done on 170 women complicated by PPROM with gestational age between $24-36^{+6}$ weeks. They were categorized into three groups Group I.24-28 weeks, Group II.28+1 to 32 weeks and Group III. $32+1$ to $36+6$ weeks. Cervical length and amniotic fluid index were measured using trans abdominal ultrasound within $24 \mathrm{hr}$ of admission. Maternal outcomes were recorded in terms of latency period, chorioamnionitis, and abruption, and neonatal outcomes were recorded in terms of birth weight, first minute APGAR score <7, NICU admission and early neonatal death. Qualitative variables were correlated using Chi-Square test /Fisher's exact test. Univariate logistic regression was used to assess cervical length and AFI as a predictor of complication. A p value of $<0.05$ was considered statistically significant.

Results: Out of 170 , majority (95) belonged to $28+1$ to 32 weeks group. Latency was inversely related to period of gestation $(\mathrm{p}<0.0001)$. A long cervical length correlated with increased latency, increased risk of chorioamnionitis and increased neonatal complications in all three groups. Also, women with PPROM having AFI $>5 \mathrm{~cm}$ had a greater mean latency period $(8.32 \pm 1.25$ days) which increased their risk of developing chorioamnionitis as compared to women with PPROM having AFI $\leq 5 \mathrm{~cm}$, who had a shorter mean latency period ( $7.63 \pm 1.07$ days) and a lower risk of developing chorioamnionitis ( $\mathrm{p}$ value $<0.0001$ ).

Conclusions: Latency is inversely related to period of gestation. A long cervical length and increased amount of AFI correlates with increased latency, increased risk of chorioamnionitis and increased neonatal complications.
\end{abstract}

Keywords: AFI, PROM, Pregnancy outcome, Cervical length

\section{INTRODUCTION}

India is the biggest contributor to the world's prematurity burden with almost 3.6 million premature births accounting for $23.6 \%$ of around 15 million global preterm births reported each year (WHO).
Preterm premature rupture of membrane (PPROM) is a breach in the chorioamniotic membrane prior to the onset of labour at $<37$ weeks of gestation. PPROM complicates $3 \%$ of all deliveries and is associated with $30-40 \%$ of preterm deliveries. It is an important risk factor for perinatal mortality and morbidity. ${ }^{1}$ 
Clinical factors associated with PPROM include low socioeconomic status, tobacco use, history of preterm labour, urinary tract infection, vaginal bleeding at any time in pregnancy, uterine distension (e.g. polyhydramnios, multifetal pregnancy), cerclage and amniocentesis. $^{2-4}$ The major maternal risks are chorioamnionitis (35\%), abruption (19\%) and sepsis $(<1 \%){ }^{3}$ Placental abruption is more common if rupture of membranes occur prior to $28 \mathrm{wk}$ of gestation. ${ }^{5}$ The risk of abruption increases $24 \mathrm{hr}$ after membrane ruptures, particularly in the presence of intrauterine infection or oligohydramnios. ${ }^{6,7}$

Chorioamnionitis is also associated with gestational age at which PPROM occurs. Major fetal morbidity is pulmonary hypoplasia, RDS, sepsis, intraventricular hemorrhage and contractures. Pulmonary hypoplasia is frequent if PPROM occurs before $26 \mathrm{wk}$ and the latency is prolonged for more than $5 \mathrm{wk}$. Latency, defined as the time from rupture of membranes till delivery has been described to be longer if PPROM occurs at an earlier gestational age. ${ }^{5,8}$

Oligohydramnios as a result of PPROM has been found to be associated with shorter latency and increased neonatal morbidity, but not associated with increased maternal or neonatal infections. ${ }^{9}$ Many studies have been conducted using CL and AFI individually to predict the perinatal outcome in PTPROM and they show poor outcome. A combination of both the parameters has not been studied yet. A quantitative analysis of the prediction of adverse pregnancy outcome using both these two parameters in this study may help in management decisions of women with PPROM.

\section{METHODS}

This was a prospective observational study conducted in Department of Obstetrics and Gynecology of a tertiary care centre in North Delhi. The study was approved by institutional ethics committee. A total of 170 women with singleton pregnancy who presented to hospital with PPROM from period of gestation 24 to $36^{+6}$ weeks were enrolled in this study. All women with PPROM were hospitalized and put on bed rest and diagnosis of PPROM was made by per speculum examination showing pooled vaginal fluid and was managed according to the hospital protocol.

Period of gestation was confirmed by accurate dating (calculated by $1^{\text {st }}$ day of last menstrual period) and ultrasound in early pregnancy if available. Patients who were in active labor, showing signs of chorioamnionitis, abruption, sepsis, with cervical cerclage operation, fetal congenital anomalies and complicated pregnancies were excluded from this study.

All diagnosed cases of PPROM were given prophylactic antibiotics for $48 \mathrm{hrs}$ (ampicillin 2 gm i.v. 6 hourly, gentamicin $2 \mathrm{mg} / \mathrm{kg}$ i.v. 8 hourly and metronidazole 500 mg i.v. 8 hourly.) Inj. Dexamethasone $6 \mathrm{mg} 12$ hourly 4 doses were given for lung maturity. Trans abdominal cervical length assessment and amniotic fluid assessment was done within 24 hours of admission. All hematological and pathological investigations were carried out and repeated weekly. No tocolytics were given. Patients were observed for sign and symptoms of chorioamnionitis i.e., maternal pyrexia, maternal tachycardia, leukocytosis, uterine tenderness, offensive vaginal discharge, and fetal tachycardia. Patients were monitored with bi-weekly TLC/DLC and HVS culture for early detection of impending infection.

Patients were monitored till they went into spontaneous labour or were induced at 34 completed weeks whichever was earlier and the outcome were recorded. Further follow up was done till the patient was discharged from the hospital for late maternal and neonatal complications. Latency was defined as the time from rupture of membranes till delivery. For the purpose of analysis, latency was stratified into three groups, 1) $\leq 2$ days, 2) 2-7 days, and 3) $>7$ days, and POG was stratified into three groups, 1) 24-28 weeks, 2) $28^{+1}-32$ weeks, and 3) $32^{+1}$ $36^{+6}$ weeks. Maternal outcomes were recorded in terms of latency period, chorioamnionitis, and abruption and neonatal outcomes were recorded in terms of birth weight, first minute APGAR score <7, NICU Admission and early neonatal death.

\section{RESULTS}

Present study reveals that latency is inversely related to period of gestation. A long cervical length correlates with increased latency, increased risk of chorioamnionitis and increased neonatal complications. As shown in Table 1, women with POG of 24-28 weeks with $\mathrm{CL} \leq 2.5 \mathrm{~cm}$ had mean latency period of $8.65 \pm 1.56$ days, $7.31 \%$ had chorioamnionitis, $24.39 \%$ babies of such women were admitted to NICU and $17.07 \%$ babies died in their early neonatal period; and women with POG of 24-28 weeks with $\mathrm{CL}>2.5 \mathrm{~cm}$ had mean latency period of $9.32 \pm 2.21$ days, $63.41 \%$ had chorioamnionitis, $75.61 \%$ babies of such women were admitted to NICU and $65.85 \%$ babies died in their early neonatal period. Similar trend of fetomaternal outcomes were also shown in the other two groups of women with POG $28^{+1}-32$ weeks and POG $32^{+1}-36^{+6}$ weeks.

As shown in Table 2, women with POG of 24-28 weeks with $\mathrm{AFI} \leq 5 \mathrm{~cm}$ had mean latency period of $7.63 \pm 1.56$ days, $19.51 \%$ had chorioamnionitis, $39.02 \%$ babies of such women were admitted to NICU and $21.95 \%$ babies died in their early neonatal period; and women with POG of 24-28 weeks with AFI $>5 \mathrm{~cm}$ had mean latency period of $8.32 \pm 2.21$ days, $51.22 \%$ had chorioamnionitis, $58.54 \%$ babies of such women were admitted to NICU and $39.02 \%$ babies died in their early neonatal period. Similar trend of feto-maternal outcomes were also shown in the other two groups of women with POG $28^{+1}-32$ weeks and POG $32^{+1}-36^{+6}$ weeks. 
Table 1: Maternal and neonatal outcome in Preterm PROM with respect to cervical length.

\begin{tabular}{|c|c|c|c|c|c|c|c|c|c|}
\hline \multirow{3}{*}{ Parameters } & \multicolumn{3}{|c|}{ POG $=24-28 w k s$} & \multicolumn{3}{|c|}{ POG $=28^{+1}-32 w k s$} & \multicolumn{3}{|c|}{ POG $=32^{+1}-36^{+6}$ wks } \\
\hline & \multicolumn{2}{|c|}{ Cervical length (cm) } & \multirow{2}{*}{ P-value } & \multicolumn{2}{|c|}{ Cervical length (cm) } & \multirow{2}{*}{ P-value } & \multicolumn{2}{|c|}{ Cervical length (cm) } & \multirow{2}{*}{ P-value } \\
\hline & $\leq 2.5$ & $>2.5$ & & $\leq 2.5$ & $>2.5$ & & $\leq 2.5$ & $>2.5$ & \\
\hline $\begin{array}{l}\text { Mean latency } \\
\text { (days) }\end{array}$ & $8.65 \pm 1.56$ & $\begin{array}{l}9.32 \\
\pm 2.21\end{array}$ & $<0.0001$ & $5.42 \pm 1.26$ & $6.14 \pm 1.46$ & $<0.0001$ & $1.36 \pm 0.32$ & $1.54 \pm 0.42$ & $<0.0001$ \\
\hline Chorioamnionitis & $3(7.31 \%)$ & $\begin{array}{l}26 \\
(63.41 \%)\end{array}$ & 0.006 & $2(2.10 \%)$ & $\begin{array}{l}20 \\
(21.05 \%)\end{array}$ & 0.006 & $1(2.94 \%)$ & $\begin{array}{l}8 \\
(23.53 \%)\end{array}$ & 0.006 \\
\hline Abruption & $0(0.00 \%)$ & $1(2.45 \%)$ & 1.000 & $0(0.00 \%)$ & $1(1.05 \%)$ & 1.000 & $1(2.94 \%)$ & $2(5.88 \%)$ & 1.000 \\
\hline Mean BW (kg) & 1.050 .5 & $1.08 \pm 0.42$ & 0.568 & $1.7 \pm 1.2$ & $1.8 \pm 1.4$ & $<0.0001$ & $2.2 \pm 0.7$ & $2.4 \pm 0.9$ & $<0.0001$ \\
\hline $\begin{array}{l}1^{\text {st }} \min \\
\text { APGAR }<7\end{array}$ & $\begin{array}{l}10 \\
(24.39 \%)\end{array}$ & $\begin{array}{l}31 \\
(75.61 \%)\end{array}$ & $<0.0001$ & $\begin{array}{l}23 \\
(24.21 \%)\end{array}$ & $\begin{array}{l}30 \\
(31.57 \%)\end{array}$ & $<0.0001$ & $2(5.88 \%)$ & $\begin{array}{l}4 \\
(11.76 \%)\end{array}$ & $<0.0001$ \\
\hline NICU admn & $\begin{array}{l}10 \\
(24.39 \%)\end{array}$ & $\begin{array}{l}31 \\
(75.61 \%)\end{array}$ & $<0.0001$ & $\begin{array}{l}23 \\
(24.21 \%)\end{array}$ & $\begin{array}{l}30 \\
(31.57 \%)\end{array}$ & $<0.0001$ & $2(5.88 \%)$ & $\begin{array}{l}4 \\
(11.76 \%)\end{array}$ & $<0.0001$ \\
\hline Early NND & $\begin{array}{l}7 \\
(17.07 \%)\end{array}$ & $\begin{array}{l}27 \\
(65.85 \%)\end{array}$ & 0.0002 & $5(5.26 \%)$ & $\begin{array}{l}17 \\
(17.89 \%)\end{array}$ & 0.0002 & $2(5.88 \%)$ & $\begin{array}{l}4 \\
(11.76 \%)\end{array}$ & 0.0002 \\
\hline
\end{tabular}

Table II: Maternal and neonatal outcome in Preterm PROM with respect to amniotic fluid index.

\begin{tabular}{|c|c|c|c|c|c|c|c|c|c|}
\hline \multirow{3}{*}{ Parameters } & \multicolumn{3}{|c|}{ POG $=24-28 w k s$} & \multicolumn{3}{|c|}{$\mathrm{POG}=28^{+1}-32 \mathrm{wks}$} & \multicolumn{3}{|c|}{ POG $=32^{+1}-36^{+6}$ wks } \\
\hline & \multicolumn{2}{|l|}{ AFI (cm) } & \multirow{2}{*}{ P-value } & \multicolumn{2}{|l|}{ AFI (cm) } & \multirow{2}{*}{ P-value } & \multicolumn{2}{|l|}{ AFI (cm) } & \multirow{2}{*}{ P-value } \\
\hline & $\leq 5$ & $>5$ & & $\leq \mathbf{5}$ & $>5$ & & $\leq \mathbf{5}$ & $>5$ & \\
\hline $\begin{array}{l}\text { Mean latency } \\
\text { (days) }\end{array}$ & $7.63 \pm 1.56$ & $8.32 \pm 2.21$ & $<0.0001$ & $5.28 \pm 1.38$ & $6.42 \pm 1.29$ & $<0.0001$ & $1.63 \pm 0.08$ & $1.85 \pm 0.05$ & $<0.0001$ \\
\hline Chorioamnionitis & $\begin{array}{l}8 \\
(19.51 \%)\end{array}$ & $\begin{array}{l}21 \\
(51.22 \%)\end{array}$ & $<0.0001$ & $7(7.36 \%)$ & $\begin{array}{l}15 \\
(15.79 \%)\end{array}$ & $<0.0001$ & $\begin{array}{l}4 \\
(11.76 \%)\end{array}$ & $\begin{array}{l}5 \\
(14.71 \%)\end{array}$ & $<0.0001$ \\
\hline Abruption & $0(0.00 \%)$ & $1(2.44 \%)$ & 1.000 & $1(1.05 \%)$ & $2(2.12 \%)$ & 1.000 & $0(0.00 \%)$ & $1(2.94 \%)$ & 1.000 \\
\hline Mean BW (kg) & $1.04 \pm 0.35$ & $1.05 \pm 0.42$ & 0.568 & $1.73 \pm 0.60$ & $1.82 \pm 0.76$ & $<0.0001$ & $2.1 \pm 0.3$ & $2.5 \pm 0.8$ & $<0.0001$ \\
\hline $\begin{array}{l}1^{\text {st }} \min \\
\text { APGAR }<7\end{array}$ & $\begin{array}{l}16 \\
(39.02 \%)\end{array}$ & $\begin{array}{l}24 \\
(58.54 \%)\end{array}$ & $<0.0001$ & $\begin{array}{l}21 \\
(22.10 \%)\end{array}$ & $\begin{array}{l}28 \\
(29.47 \%)\end{array}$ & $<0.0001$ & $\begin{array}{l}4 \\
(11.76 \%)\end{array}$ & $\begin{array}{l}7 \\
(20.59 \%)\end{array}$ & $<0.0001$ \\
\hline NICU admn & $\begin{array}{l}16 \\
(39.02 \%)\end{array}$ & $\begin{array}{l}24 \\
(58.54 \%)\end{array}$ & $<0.0001$ & $\begin{array}{l}21 \\
(22.10 \%)\end{array}$ & $\begin{array}{l}28 \\
(29.47 \%)\end{array}$ & $<0.0001$ & $\begin{array}{l}4 \\
(11.76 \%)\end{array}$ & $\begin{array}{l}7 \\
(20.59 \%)\end{array}$ & $<0.0001$ \\
\hline Early NND & $\begin{array}{l}9 \\
(21.95 \%)\end{array}$ & $\begin{array}{l}16 \\
(39.02 \%)\end{array}$ & 0.0002 & $\begin{array}{l}14 \\
(14.73 \%)\end{array}$ & $\begin{array}{l}16 \\
(16.84 \%)\end{array}$ & 0.0002 & $3(8.82 \%)$ & $\begin{array}{l}4 \\
(11.76 \%)\end{array}$ & 0.0002 \\
\hline
\end{tabular}

\section{DISCUSSION}

The finding of the present study that longer the cervix, more is the latency period, is consistent with Rizzo et al who examined 92 women and reported that the median interval to delivery was 2 days when cervical length was $<20 \mathrm{~mm}$, compared to 6 days with longer cervix. ${ }^{10}$ Similarly, in a recent study by Mehra et al a shorter TVCL independently predicted delivery within 7 days in women presenting with PPROM and TVCL $>2 \mathrm{~cm}$ greatly improved the potential to remain undelivered at 7 days following cervical length assessment. ${ }^{11}$ In contrast, Carlan et al found no significant difference in latency period based on cervical length. ${ }^{12}$

In this study, women with AFI $>5 \mathrm{~cm}$ in all the three groups had higher mean latency period as compared to women with AFI $\leq 5 \mathrm{~cm}$ ( $\mathrm{p}$ value $<0.0001$ ). This finding was consistent with study of Tavassoli $F$ et al who demonstrated that patients with AFI $<5$ exhibited a significantly shorter latency period $(\mathrm{p}=0.049) .{ }^{13} 43 \%$ of cases delivered during the first 48 hours and only $6.6 \%$ of pregnancies were prolonged by more than 2 weeks. However, in group with $(\mathrm{AFI} \geq 5), 31.7 \%$ of patients delivered during the first 48 hours and $21.7 \%$ of pregnancies were prolonged by more than 2 weeks. Most studies have not reported this point, but in a study by Borna et al the latency period was observed to be equal in both groups. ${ }^{14}$ Piazze et al and Vermillion et al reported significant correlation between oligohydramnios and latency period. ${ }^{15,16}$ Mehra S also found that an AFI $<5 \mathrm{~cm}$ independently predicted delivery within 7 days in women presenting with PPROM. ${ }^{11}$

Present study also shows that longer the cervix and larger the AFI, more was the incidence of maternal infection, however the literature is conflicting in the correlation of AFI with occurrence of chorioamnionitis in cases of PPROM. Mercer et al and Piazze et al found no relationship between chorioamnionitis and oligohydramnios while Borna et al and Moberg et al found a significant correlation between $\mathrm{AFI}<5$ and $\mathrm{a}$ higher rate of chorioamnionitis. ${ }^{9,14,15,17}$ In the present study, the incidence of chorioamnionitis was higher in 
women with $\mathrm{CL}>2.5 \mathrm{~cm}$ and AFI $>5 \mathrm{~cm}$. This increased incidence of chorioamnionitis can be probably due to the lower socioeconomic strata of patients coming to our hospital making this difference with an increased incidence of chorioamnionitis with longer latency.

Women presenting with preterm PROM are at increased risk of developing abruption, risk being higher in the presence of oligohydramnios especially within 24 hours of rupture of membrane. Present study however shows no significant correlation between abruption, AFI and CL consistent with study by CC Ananth et al. Compared women with intact membranes, the relative risk for abruption among preterm PROM and whose membranes were ruptured for 24-47 hours and 48 hours or more before delivery, respectively, were 2.37 (95\%CI 0.99 9.09), and 9.87 (95\% CI 3.57-27.82). ${ }^{7}$ When preterm PROM was accompanied by intrauterine infections, the RR for abruption was 9.03 (95\%CI 2.80-29.15) compared with women with intact membranes and no infections. Similarly, preterm PROM accompanied by oligohydramnios conferred over a 7.17 -fold risk $(95 \%$ CI 1.35-38.10) for abruption compared with women with neither of these 2 conditions.

Regarding neonatal outcome, we found that there was no significant relation between mean birth weight, CL and AFI which is consistent with the study of Tavasolli et al. ${ }^{13}$ Present study also shows that longer the cervix and larger the AFI, more was the latency, more was the risk of maternal infection and this also had an impact on fetal outcome in the form of $1^{\text {st }}$ minute APGAR $<7$, NICU admission and early NND. Tavasolli et al found that first minute and five minute Apgar score $\leq 7$ was significantly higher in group with AFI $<5$, however the difference was not significant between the two groups. ${ }^{13}$ In contrast Piazze et al found that there was a significant association between five minute Apgar score $\leq 7$ and AFI $<5$ ( $p$ $<0.001$ ), but in this study, the association was not statistically significant. ${ }^{15}$ Present study is contradictory to Tavasolli et al which found that the overall rate of early neonatal death was $8.8 \% .{ }^{13} 10$ cases $(13.2 \%)$ of neonatal deaths were observed in group with AFI $<5$ while only 2 cases $(3.3 \%)$ were recorded for group with AFI $\geq 5$. Therefore, the rate of neonatal death was significantly higher in group with AFI $<5$ compared to group with AFI $\geq 5$ with a $\mathrm{p}$ value of 0.045 .

\section{CONCLUSION}

Latency is inversely proportional to period of gestation which means lesser the period of gestation more will be the latency period. A long cervical length and increased amount of amniotic fluid index co-relates with increased incidence of chorioamnionitis and neonatal complications probably due to increased latency. Since the latency cannot be predicted in advance in pre-term pre-mature rupture of membranes, women with shorter cervix and lesser amniotic fluid index needs to be hospitalized and managed aggressively and should be monitored vigorously for further complications. The women with longer cervix and higher amniotic fluid may require a longer duration of antibiotic coverage since the latency period is more.

\section{Funding: No funding sources}

Conflict of interest: None declared

Ethical approval: The study was approved by the Institutional Ethics Committee

\section{REFERENCES}

1. Blott M, Greenough A. Neonatal outcome after prolonged rupture of the membranes starting in the second trimester. Arch Dis Child. 1988;63:1146-50.

2. ACOG Practice bulletin no 1. Premature rupture of membranes. Clinical management guidelines for obstetrician-gynecologists. Int J Gynaecol Obstet. 1998;63(1):75-84.

3. Mercer B, Milluzzi C, Collin M. Periviable birth at 20 to 26 wk of gestation: proximate causes, previous obstetric history and recurrence risk. Am J Obstet Gynecol. 2005 Sep;193(3 Pt 2):1175-80.

4. Waters TP, Mercer BM. The management of preterm premature rupture of the membranes near the limit of fetal viability. Am J Obstet Gynecol. 2009 Sep;201(3):230-40.

5. ACOG Practice Bulletins No 80: premature rupture of membranes. Obstet Gynaecol. 2007;109(4):100719.

6. Gonan R, Hannah ME, Milligan JE. Does prolonged premature rupture of the membranes predispose to abruption placenta? Obstet Gynecol. 1989;74:34750.

7. Ananth CC, Oyelese $\mathrm{Y}$, Srinivas $\mathrm{N}$, Yeo L, Vintzileos AM. Preterm premature rupture of membranes, intrauterine infection, and oligohydramnios: risk factors for placental abruption. Obstet Gynecol. 2004 Jul;104(1):71-7.

8. Mercer BM. Preterm premature rupture of the membranes. Obstet Gynecol. 2003;101(1):178-93.

9. Mercer BM, Rabello YA, Thumau GR, Modovnik M, Goldenberg RL, Das AF et al. The NICHDMFMU antibiotic treatment of preterm PROM study: Impact of initial amniotic fluid volume on pregnancy outcome. Am J Obstet Gynecol. 2006 Feb;194(2):438-45.

10. Rizzo G, Capponi A, Angelini E, Vlachopoulou A, Grassi C, Romanini C. The value of transvaginal ultrasonographic examination of the uterine cervix in predicting preterm delivery in patients with preterm premature rupture of membranes. Ultrasound Obstet Gynecol. 1998;11:23-9.

11. Mehra S, Amon E, Hopkins S, Gavard JA, Shyken J. Transvaginal cervical length and amniotic fluid index: can it predict delivery latency following preterm premature rupture of membranes? Am J Obstet Gynecol. 2015 Mar;212(3):400.e1-9.

12. Carlan SJ, Richmond LB, O'Brien WF. Randomized trial of endovaginal ultrasound in preterm premature 
rupture of membranes. Obstet Gynecol. 1997;89:458-61.

13. Tavassoli F, Ghasemi M, Mohamadzade A, Sharifian J, Survey of pregnancy outcome in preterm premature rupture of membranes with amniotic fluid index $<5$ and $\geq 5$. Oman Med J. 2010;25(2):118-23.

14. Borna S, Borna H, Khazardoost S, Hantoushzadeh S. Perinatal outcome in preterm premature rupture of membranes with Amniotic fluid index <5 (AFI<5). BMC Pregnancy Childbirth. 2004 Aug;4(1):15.

15. Piazze J, Anceschi M, Cerekja A. Validity of amniotic fluid index in preterm rupture of membranes. J Perinat Med. 2007;35(5):394-8.

16. Vermillion ST, Kooba AM, Soper DE. Amniotic Fluid index value after preterm premature rupture of membrane and subsequent perinatal infection. Am J Obstet Gynecol. 2000;183(2):271-6.

17. Moberg IJ, Garete TJ, Freeman RK. Fetal heart rate patterns of fetal distress in patient with preterm premature rupture of membranes. Obstet Gynecol. 1984 Jul;64(1):60-4.

Cite this article as: Patil P, Kumari A, Anand HP. Ultrasonographic evaluation of cervical length and amniotic fluid index as predictor of pregnancy outcome in case of preterm premature rupture of membrane. Int J Reprod Contracept Obstet Gynecol 2018;7:1411-5. 